\title{
Herbicidas aplicados em pré-emergência para o controle de Ipomoea spp. na cultura de cana-de-açúcar em época seca ${ }^{1}$
}

\section{Herbicides applied in pre-emergence to control Ipomoea spp. in sugarcane in dry}

season

\begin{abstract}
Roberto Estevão Bragion Toledo ${ }^{2}$; Antonio Carlos da Silva Junior ${ }^{3}$; Raphael Mereb Negrisoli ${ }^{4}$;
\end{abstract} Eduardo Negrisoli ${ }^{5}$; Marcelo Rocha Corrêa ${ }^{6}$; Murillo Grespan da Rocha ${ }^{7}$; Ricardo Victória Filho ${ }^{8}$

Resumo - O controle de plantas daninhas por meio da aplicação de herbicidas de longa atividade residual é importante estratégia para a supressão destas em diferentes épocas do ano. $\mathrm{O}$ objetivo deste trabalho foi avaliar a atividade residual de herbicidas, aplicados em pré-emergência da canade-açúcar, sobre o controle de corda-de-viola na época seca, e a seletividade dos herbicidas a cultura da cana-de-açúcar. $\mathrm{O}$ experimento foi realizado no delineamento experimental de blocos casualizados, com quatro repetições. Os tratamentos foram $\left(\mathrm{g}\right.$ i.a. ha $\left.{ }^{-1}\right)$ : diuron + hexazinone + sulfometuron $(1.025,1+289+24,65)$; amicarbazone (1.050); amicarbazone e aplicação sequencial de isoxaflutole (700 e 60); sulfentrazone (800); imazapic (133); tebuthiuron e aplicação sequencial de isoxaflutole (750 e 60), e uma testemunha sem aplicação de herbicidas. Os herbicidas diuron + hexazinone + hexazinone e amicarbazone causaram níveis de controle de Ipomoea hederifolia de $96 \%$ e $92,5 \%$, respectivamente. Já para a Ipomoea quamoclit, todos os herbicidas estudados proporcionaram excelentes níveis de controle. Para a Ipomoea nil, os herbicidas diuron + hexazinone + sulfometuron, amicarbazone, amicarbazone e isoxaflutole e o sulfentrazone apresentaram níveis de controle de muito bons a excelente, enquanto que o herbicida tebuthiuron e isoxaflutole apenas bom nível de controle $(88,8 \%)$ e o imazapic com nível insatisfatório de controle $(45 \%)$. Portanto, pode-se concluir que os herbicidas diuron + hexazinone + sulfometuron $\left(1025+289+24,65\right.$ g i.a. ha $\left.{ }^{-1}\right)$, amicarbazone $\left(1050\right.$ g i.a. ha $\left.{ }^{-1}\right)$, sulfentrazone $\left(800 \mathrm{~g} \mathrm{i}^{\left.\mathrm{a} . \mathrm{ha}^{-1}\right), \mathrm{e}}\right.$ as aplicações sequenciais de amicarbazone (700 g i.a. ha $\left.{ }^{-1}\right)$ e isoxaflutole $\left(60 \mathrm{~g}\right.$ i.a. ha $\left.\mathrm{ha}^{-1}\right)$, podem ser considerados como excelentes alternativas para o controle de diferentes espécies de corda-deviola (I. hederifolia, I. quamoclit e I. nil), enquanto que o herbicida imazapic (133 $\mathrm{g}$ i.a. ha $\left.{ }^{-1}\right)$ e a aplicação sequencial de tebuthiuron $\left(750 \mathrm{~g}\right.$ i.a. ha $\left.{ }^{-1}\right)$ e isoxaflutole $\left(60 \mathrm{~g}\right.$ i.a. ha $\left.{ }^{-1}\right)$ podem ser considerados como alternativas para o controle apenas de Ipomoea quamoclit quando aplicados em

\footnotetext{
${ }^{1}$ Recebido para publicação em 07/12/2015 e aceito em 13/02/2016.

${ }^{2}$ ESALQ/USP. Av. Pádua Dias, 11, 13418-900. Piracicaba, SP. E-mail: roberto.e.toledo@ gmail.com.

3 FCAV/UNESP. Via de Acesso Prof. Paulo Donato Castellane s/n 14884-900. Jaboticabal, SP. E-mail: acsjr_agro@hotmail.com.

${ }^{4}$ FEIS/UNESP. Av. Brasil, 56, 15385-000. Ilha Solteira, SP. E-mail: raphamereb@ hotmail.com.

5 Techfield Assessoria e Consultoria Agrícola. Rod. Marechal Rondon, km 253, 18608-970. Botucatu, SP. E-mail: eduardo.negrisoli@techfield.agr.br.

${ }^{6}$ Techfield Assessoria e Consultoria Agrícola. Rod. Marechal Rondon, km 253, 18608-970. Botucatu, SP. E-mail: marcelo.r.correa@techfield.agr.br.

7 Techfield Assessoria e Consultoria Agrícola. Rod. Marechal Rondon, km 253, 18608-970. Botucatu, SP. E-mail: mugrespan@yahoo.com.

${ }^{8}$ ESALQ/USP. Av. Pádua Dias, 11, 13418-900. Piracicaba, SP. E-mail: rvictori@usp.br.
} 
pré-emergência da cultura da cana-de-açúcar na época seca.

Palavras-chaves: controle residual; Ipomoea nil; Ipomoea hederifolia; Ipomoea quamoclit

\begin{abstract}
The weed control through the application of long residual activity of herbicides is important strategy for the removal of these at different times of the year. The objective of this study was to evaluate the residual activity of herbicides applied pre-emergence of sugarcane on the morning glory of control in the dry season, and the selectivity of the herbicides sugarcane crop. The experiment was conducted in a randomized block design with four replications. The treatments were $\left(\mathrm{g}\right.$ a.i. $\left.\mathrm{ha}^{-1}\right)$ : hexazinone + diuron + sulfometuron $(1,025.1+289+24.65)$; Amicarbazone $(1,050)$; amicarbazone and sequential application of isoxaflutole (700 and 60); sulfentrazone (800); imazapic (133); tebuthiuron and sequential application of isoxaflutole (750 and 60), and a control without herbicide application. The hexazinone + diuron + hexazinone and amicarbazone caused Ipomoea hederifolia control levels of $96 \%$ and $92.5 \%$, respectively. As for the Ipomoea quamoclit, all herbicides studied provided excellent levels of control. For Ipomoea nil, the herbicides hexazinone + diuron + sulfometuron, amicarbazone, amicarbazone and isoxaflutole and sulfentrazone control showed very good to excellent, while tebuthiuron and isoxaflutole herbicides only good level of control (88.8\%) and imazapic with unsatisfactory level of control (45\%). Therefore, it can be concluded that hexazinone + diuron + sulfometuron $(1,025+289+24.65 \mathrm{~g}$ a.i. $\left.\mathrm{ha}^{-1}\right)$, amicarbazone $\left(1050 \mathrm{~g}\right.$ a.i. $\left.\mathrm{ha}^{-1}\right)$, sulfentrazone $\left(800 \mathrm{~g}\right.$ a.i. ha $\left.{ }^{-1}\right)$, and the sequential applications amicarbazone (700 $\mathrm{g}$ a.i. $\left.\mathrm{ha}^{-1}\right)$ and isoxaflutole $\left(60 \mathrm{~g}\right.$ a.i. ha $\left.{ }^{-1}\right)$, they can be considered as excellent alternatives for the control of different morning glory species (I. hederifolia, $I$. quamoclit and $I$. nil), while the imazapic (133 $\mathrm{g}$ a.i. $\left.\mathrm{ha}^{-1}\right)$ and the sequential application of tebuthiuron $\left(750 \mathrm{~g}\right.$ a.i. $\left.\mathrm{ha}^{-1}\right)$ and isoxaflutole $\left(60 \mathrm{~g}\right.$ a.i. $\left.\mathrm{ha}^{-1}\right)$ can be considered as alternatives to control only Ipomoea quamoclit when applied in pre emergence of sugarcane crop in the dry season.
\end{abstract}

Keywords: residual control; Ipomoea nil; Ipomoea hederifolia; Ipomoea quamoclit

\section{Introdução}

Um dos pontos mais críticos no processo produtivo da cana-de-açúcar (Saccharum spp.) é a capacidade de interferência no desenvolvimento e na produtividade imposta pelas plantas daninhas (Pitelli, 1995; Kuva et al., 2008; Toledo e Negrisoli, 2011; Jones e Griffin, 2009). A intensidade de interferência entre uma cultura agrícola e comunidade infestante depende de fatores ligados à própria cultura, como a variedade, espaçamento e densidade de plantio, fatores ligados à comunidade infestante, como composição específica, densidade e distribuição dos indivíduos na cultura e da época e extensão do período em que a cultura da cana-de-açúcar e a comunidade infestante estiveram em convivência (Pitelli, 1995).
Há vários fatores que influenciam o controle das plantas daninhas, tanto na época chuvosa ou seca do ano, sendo a umidade, teores de argila, matéria orgânica ou pH, assim como as características físico-químicas dos herbicidas, como a solubilidade e a pressão de vapor, os mais importantes (Walker et al., 1992). Pode ocorrer de esses fatores intervirem nos processos de lixiviação, adsorção e degradação biológica, afetando a disponibilidade e persistência dessas moléculas herbicidas na solução do solo, consequentemente interferindo na absorção das mesmas pelas raízes das plantas daninhas e na eficiência do herbicida (Silva Junior et al., 2015a; Silva Junior et al., 2015b; Walker et al., 1992)

O controle de plantas daninhas utilizando-se de herbicidas pré-emergentes, com 
alta atividade residual, é um dos fatores com grande eficiência, devido a maior supressão das plantas daninhas durante o período crítico de competição da cultura (Esquivel et al., 2010; Mancuso et al., 2011; Miller e Westra, 1998).

Como alternativa para o controle químico de plantas daninhas em cana-de-açúcar na época seca, Toledo et al. (2010) destacam excelentes resultados da associação dos herbicidas diuron + hexazinone + sulfometuron no controle de várias espécies de corda-de-viola (Ipomoea hederifolia, Merremia cissoides, Ipomoea grandifolia, Ipomoea quamoclit e Ipomoea nil), bem como excelente controle de gramíneas (Brachiaria decumbens, $B$. plantaginea, $P$. maximum e Digitaria sp.).

Dentro deste contexto, o objetivo deste trabalho foi avaliar a atividade residual de herbicidas, aplicados em pré-emergência da cana-de-açúcar, sobre o controle de corda-deviola na época seca, e a seletividade dos herbicidas a cultura da cana-de-açúcar.

\section{Material e Métodos}

$\mathrm{O}$ experimento foi conduzido em uma área comercial de cana-de-açúcar, soqueira de segundo corte recém-colhida manualmente com despalha pelo fogo, em solo com textura areia franca (EMBRAPA, 2013), localizada na região de Monte Alto (SP). Os dados de precipitação durante o período de condução do experimento estão apresentados na Figura 1.

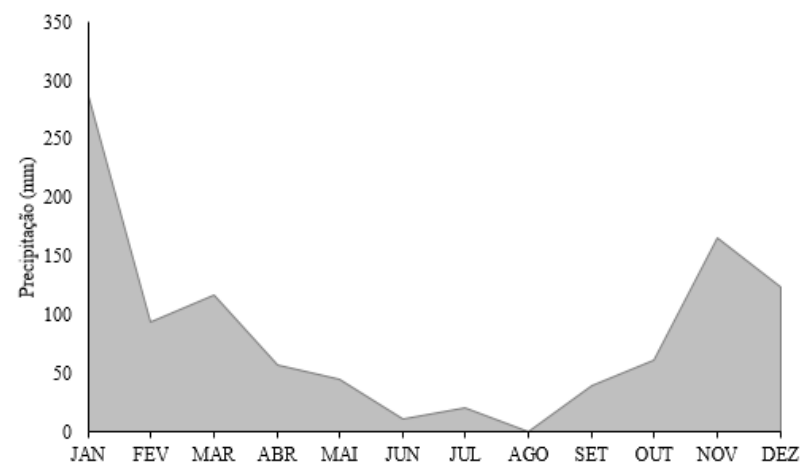

Figura 1. Dados de precipitação pluvial (mm) ocorridas durante o período de desenvolvimento do presente trabalho. Monte Alto (SP), 2012.
A variedade presente na área era a CTC 2, plantada no espaçamento de 1,4 m entrelinhas. As plantas daninhas estudadas (Ipomoea hederiofolia, Ipomoea quamoclit $\mathrm{e}$ Ipomoea nil) foram semeadas nas respectivas parcelas na proporção para que houvesse uma infestação uniforme de 50 plantas $\mathrm{m}^{-2}$.

$\mathrm{O}$ delineamento experimental adotado foi o de blocos casualizados, com quatro repetições. As parcelas tiveram como dimensões 6 metros de largura por 8 metros de comprimento, totalizando uma área de $48 \mathrm{~m}^{2}$. Entretanto, como área útil, a área foi de $42 \mathrm{~m}^{2}$, considerando a região central de cada parcela.

Os tratamentos aplicados foram: diuron/hexazinone/sulfometuron

$1.025,1 / 289,0 / 24,65$ g ha $^{-1} \quad\left(\right.$ Front $^{\circledR}$, 603/170/14,5 $\mathrm{g} \mathrm{kg}^{-1}$ de i. a., WG, Dupont); amicarbazone $-1.050,0 \mathrm{~g} \mathrm{ha}^{-1}\left(\right.$ Dinamic $^{\circledR}, 700 \mathrm{~g}$ $\mathrm{kg}^{-1}$ de i.a. WG, Arysta LifeScience); amicarbazone - 700,0 g ha ${ }^{-1}$ e aplicação sequencial de isoxaflutole - 60,0 $\mathrm{g} \mathrm{ha}^{-1}$ (Provence $750 \mathrm{WG}^{\circledR}, 750 \mathrm{~g} \mathrm{~kg}^{-1}$ de i. a., WG, Bayer); sulfentrazone - 800,0 $\mathrm{g} \mathrm{ha}^{-1}$ (Boral 500 $\mathrm{SC}^{\circledR}, 500 \mathrm{~g} \mathrm{~L}^{-1}$ de i. a., SC, FMC); imazapic 133,0 g ha $^{-1}$ (Plateau $^{\circledR}, 700 \mathrm{~g} \mathrm{~kg}^{-1}$ de i. a., WG, Basf); tebuthiuron - $750 \mathrm{~g} \mathrm{ha}^{-1}$ (Combine 500 $\mathrm{SC}^{\circledR}, 500 \mathrm{~g} \mathrm{~L}^{-1}$ de i. a., SC, Dow AgroSciences) e aplicação sequencial de isoxaflutole - 60,0 g $\mathrm{ha}^{-1}$, e uma testemunha sem aplicação de herbicidas. $\mathrm{O}$ isoxaflutole foi aplicado em sequência aos herbicidas descritos nos tratamentos no qual ele se encontra. A aplicação de todos os herbicidas foi realizada em préemergência total tanto das plantas daninhas quanto da cultura da cana-de-açúcar na época seca (01/07/2012). Para tanto, foi utilizado um pulverizador costal pressurizado por $\mathrm{CO}_{2}$, a uma pressão constante de $200,0 \mathrm{kPa}$, provida de seis pontas Teejet TTi 110.02 , espaçados de $0,50 \mathrm{~m}$ e taxa de aplicação de $200 \mathrm{~L} \mathrm{ha}^{-1}$.

As avaliações visuais de controle das plantas daninhas e fitotoxicidade na cultura da cana-de-açúcar foram realizadas aos 30, 60 e 90 dias após a aplicação (DAA), atribuindo-se notas visuais em percentual, no qual $0 \%$ representou ausência de controle e $100 \%$ morte 
das plantas daninhas, e para fitotoxicidade $0 \%$ representou ausência de toxicidade ou injúria à cultura, e $100 \%$ para a morte das plantas de cana-de-açúcar (SBCPD, 1995).

Para efeito de comparações, os dados foram submetidos à análise de variância pelo teste $\mathrm{F}$ e as médias comparadas pelo teste de Tukey ao nível de 5\% de probabilidade com auxílio do programa estatístico Sisvar 5.1 (Ferreira, 2011).

\section{Resultados e Discussão}

Pode-se observar na Tabela 1, que aos 30 dias após a aplicação (30 DAA) que a formulação comercial de diuron + hexazinone + sulfometuron, amicarbazone, a aplicação sequencial de amicarbazone e isoxaflutole, a formulação de sulfentrazone e aplicação sequencial de tebuthiuron $\mathrm{e}$ isoxaflutole apresentaram excelentes resultados de controle (96,3 a 100\%) para a Ipomoea hederifolia, enquanto que o herbicida imazapic resultou apenas em um nível regular de controle (77,5\%).

Já a partir dos 60 e até os 90 DAA notase que os níveis de controle para esta espécie de corda-de-viola foram reduzindo ao longo do tempo, destacando-se assim os tratamentos com os herbicidas diuron + hexazinone + sulfometuron com excelente controle $(97,5$ a $96,0 \%$ ), amicarbazone com um nível muito bom de controle (91,3 a 92,5\%), tratamentos com maior efeito ou atividade residual sendo superiores quando comparados aos níveis de controle proporcionados pelo o herbicida sulfentrazone $(88,8$ a $86,3 \%)$ e as aplicações sequenciais de amicarbazone e isoxaflutole $(91,3$ a $88,8 \%)$ ou tebuthiuron e isoxaflutole $(93,8$ a $87,5 \%)$.

Tabela 1. Valores médios (\%) do controle de Ipomoea hederiofolia por herbicidas aplicados em pré-emergência na época seca. Monte Alto (SP), 2012.

\begin{tabular}{|c|c|c|c|}
\hline \multirow{2}{*}{ Tratamentos } & \multicolumn{3}{|c|}{ I. hederifolia } \\
\hline & $30 \mathrm{DAA}^{2}$ & $60 \mathrm{DAA}$ & $90 \mathrm{DAA}$ \\
\hline diuron + hexazinone + sulfometuron & $100,0 \mathrm{a}$ & $97,5 \mathrm{a}$ & $96,0 \mathrm{a}$ \\
\hline amicarbazone & $96,3 \mathrm{ab}$ & $91,3 \mathrm{ab}$ & $92,5 \mathrm{a}$ \\
\hline amicarbazone + isoxaflutole ${ }^{1}$ & $100,0 \mathrm{a}$ & $91,3 \mathrm{ab}$ & $88,8 \mathrm{ab}$ \\
\hline sulfentrazone & $100,0 \mathrm{a}$ & $88,8 \mathrm{ab}$ & $86,3 \mathrm{ab}$ \\
\hline imazapic & $77,5 \mathrm{~b}$ & $80,0 \mathrm{~b}$ & $75,0 \mathrm{~b}$ \\
\hline tebuthiuron + isoxaflutole ${ }^{1}$ & $98,8 \mathrm{ab}$ & $93,8 \mathrm{a}$ & $87,5 \mathrm{ab}$ \\
\hline $\bar{F}$ & $2,71 *$ & $3,13^{*}$ & $3,11 *$ \\
\hline $\mathrm{CV}(\%)$ & 9,24 & 5,52 & 6,79 \\
\hline D.M.S. & 21,31 & 11,62 & 13,97 \\
\hline
\end{tabular}

${ }^{1}$ Associação dos herbicidas em aplicação sequencial em pré-emergência da cultura e das plantas daninhas. ${ }^{2}$ Dias após a aplicação. Médias seguidas de mesma letra, nas colunas, não diferem entre si, pelo teste de Tukey ( $>00,05)$. *Significativo a $5 \%$ de probabilidade

Os excelentes resultados de controle obtidos com a formulação comercial de diuron + hexazinone + sulfometuron no controle de Ipomoea hederifolia pode ter ocorrido possivelmente devido ao residual prolongado dos herbicidas inibidores de fotossistema II e o respectivo posicionamento no solo do diuron e hexazinone, somados ainda às características do sulfometuron (Toledo e Negrisoli, 2011). Por outro lado, os resultados observados no presente trabalho com o herbicida amicarbazone (inibidor de FSII) foram semelhantes aos obtidos por Carvalho et al. (2011) em estudo de diferentes doses do herbicida amicarbazone para o manejo de I. hederifolia.

Azania et al. (2009) observaram controle positivo sobre plantas daninhas do gênero Ipomoea com o uso dos herbicidas amicarbazone e sulfentrazone, isolados ou não, porém com controle insatisfatório do herbicida imazapic sobre estas espécies de plantas, o que pode estar associado não somente ao mecanismo de ação dos herbicidas amicarbazone (inibidor de fotossistema II), sulfentrazone (inibidor de protox) e imazapic 
(inibidor de ALS), mas sim ao espectro de ação de controle.

Já para a espécie de corda-de-viola Ipomoea quamoclit, pode-se observar durante todo o período experimental que a formulação comercial de diuron + hexazinone + sulfometuron, amicarbazone, sulfentrazone e as aplicações sequenciais de amicarbazone e isoxaflutole, e/ou de tebuthiuron e isoxaflutole proporcionaram excelentes níveis de controle $(99,5$ a 100\%), sendo superiores estatisticamente ao nível de controle apresentado pelo herbicida imazapic, considerado como muito bom $(93,8 \%)$ (Tabela 2).

Tabela 2. Valores médios (\%) do controle de Ipomoea quamoclit por herbicidas aplicados em préemergência na época seca. Monte Alto (SP), 2012.

\begin{tabular}{|c|c|c|c|}
\hline \multirow{2}{*}{ Tratamentos } & \multicolumn{3}{|c|}{ I. quamoclit } \\
\hline & $30 \mathrm{DAA}^{2}$ & $60 \mathrm{DAA}$ & 90 DAA \\
\hline $\begin{array}{l}\text { diuron + hexazinone + sulfometuron } \\
\end{array}$ & $100,0 \mathrm{a}$ & $100,0 \mathrm{a}$ & $100,0 \mathrm{a}$ \\
\hline amicarbazone & $100,0 \mathrm{a}$ & $100,0 \mathrm{a}$ & $100,0 \mathrm{a}$ \\
\hline amicarbazone + isoxaflutole $^{1}$ & $100,0 \mathrm{a}$ & $100,0 \mathrm{a}$ & $100,0 \mathrm{a}$ \\
\hline sulfentrazone & $99,5 \mathrm{a}$ & $99,5 \mathrm{a}$ & $99,5 \mathrm{a}$ \\
\hline imazapic & $85, .0 \mathrm{a}$ & 93,8 a & $93,8 \mathrm{~b}$ \\
\hline tebuthiuron + isoxaflutole ${ }^{1}$ & $99,5 \mathrm{a}$ & $100,0 \mathrm{a}$ & $100,0 \mathrm{a}$ \\
\hline F & $2,07^{\text {ns }}$ & $1,61^{\mathrm{ns}}$ & $5,96 *$ \\
\hline $\mathrm{CV}(\%)$ & 6,97 & 3,24 & 1,68 \\
\hline D.M.S. & 16,27 & 7,63 & 3,97 \\
\hline
\end{tabular}

${ }^{1}$ Associação dos herbicidas em aplicação sequencial em pré-emergência da cultura e das plantas daninhas. ${ }^{2}$ Dias após a aplicação. Médias seguidas de mesma letra, nas colunas, não diferem entre si, pelo teste de Tukey ( $>>0,05)$. *Significativo a $5 \%$ de probabilidade; ${ }^{\text {ns }}$ não significativo

Santos et al. (2009), estudando o herbicida imazapic isolado ou com associações, registraram controle de Ipomoea grandifolia aos 20 DAA de $62 \%$, a partir dos 35 DAA o controle desta planta daninha evoluiu para níveis superiores à 92\%. Silva et al. (2011) observaram um controle regular a bom para Ipomoea triloba $(<89 \%)$ quando aplicado imazapic diretamente ao solo na dose de $300 \mathrm{~g} \mathrm{ha}^{-1}$, em préemergência da planta daninha, porém sua ação pode ter sido prejudicada pelo excesso de umidade do solo. Já nos resultados obtidos neste trabalho, a eficácia do herbicida imazapic foi inferior, o que provavelmente é explicado em função da época de aplicação, dose, espécie de corda-de-viola e nível de infestação.

Para a Ipomoea nil, os herbicidas diuron + hexazinone + sulfometuron, amicarbazone, amicarbazone, sulfentrazone e a aplicação sequencial de amicarbazone e isoxaflutole foram os tratamentos que apresentaram os maiores níveis de controle, considerados de muito bons (>90 a 95\%) a excelente (>96 a $100 \%$ ) durante todo o período experimental (30 aos 90 DAA), enquanto que o herbicida tebuthiuron e isoxaflutole resultaram um controle muito bom $(93,3 \%)$ no período de 30 a 60 DAA e apenas bom nível de controle $(88,8 \%)$ aos 90 DAA. Já o herbicida imazapic não proporcionou bons níveis de controle, sendo regular a insatisfatório dos 30 aos 90 DAA (61,3 a 45\%), apresentando diferenças significativas e estatísticas quando comparado aos demais tratamentos herbicidas (Tabela 3).

Em relação à fitotoxicidade dos tratamentos herbicidas estudados, observa-se que os sintomas foram leves, caracterizados por cloroses nas folhas da cana-de-açúcar até os 30 DAA para o tratamento com os herbicidas amicarbazone e isoxaflutole e até os 60 DAA para o herbicida imazapic, mesmo quando aplicados em pré-emergência total da cultura da cana-de-açúcar (Tabela 4). O mesmo comportamento de fitotoxidade também foi observado por Azania et al. (2001) quando aplicado imazapic (60 DAA) quando houve restrição hídrica na região, diminuindo este efeito com a regularidade das chuvas. 
Tabela 3. Valores médios (\%) do controle de Ipomoea nil por herbicidas aplicados em préemergência na época seca. Monte Alto (SP), 2012.

\begin{tabular}{|c|c|c|c|}
\hline \multirow{2}{*}{ Tratamentos } & \multicolumn{3}{|c|}{ I. $n i l$} \\
\hline & $30 \mathrm{DAA}^{2}$ & $60 \mathrm{DAA}$ & 90 DAA \\
\hline diuron + hexazinone + sulfometuron & $98,3 \mathrm{a}$ & $96,8 \mathrm{a}$ & $96,3 \mathrm{a}$ \\
\hline amicarbazone & $97,5 \mathrm{a}$ & $95,3 \mathrm{a}$ & $93,5 \mathrm{a}$ \\
\hline amicarbazone + isoxaflutole ${ }^{1}$ & $100,0 \mathrm{a}$ & $100,0 \mathrm{a}$ & $99,0 \mathrm{a}$ \\
\hline sulfentrazone & 99,5 a & $99,5 \mathrm{a}$ & $95,3 \mathrm{a}$ \\
\hline imazapic & $61,3 \mathrm{~b}$ & $60,0 \mathrm{~b}$ & $45,0 \mathrm{~b}$ \\
\hline tebuthiuron + isoxaflutole ${ }^{1}$ & 93,3 a & $93,3 \mathrm{a}$ & $88,8 \mathrm{a}$ \\
\hline $\mathrm{F}$ & $10,39 *$ & $28,02 *$ & $37,26^{*}$ \\
\hline $\mathrm{CV}(\%)$ & 8,16 & 5,11 & 6,20 \\
\hline D.M.S. & 18,13 & 11,2 & 13,08 \\
\hline
\end{tabular}

Já para os herbicidas diuron + isoxaflutole não foram observadas nenhum hexazinone + sulfometuron, amicarbazone e a sintoma de fitotoxicidade para a cultura da canaaplicação sequencial de tebuthiuron e de-açúcar até os 90 DAA.

Tabela 4. Valores médios (\%) de fitotoxicidade causado pelos herbicidas na cultura da cana-deaçúcar cultivada em solo arenoso. Monte Alto (SP), 2012.

\begin{tabular}{|c|c|c|c|}
\hline \multirow{2}{*}{ Tratamentos } & \multicolumn{3}{|c|}{ Fitotoxicidade } \\
\hline & $30 \mathrm{DAA}^{2}$ & $60 \mathrm{DAA}$ & 90 DAA \\
\hline diuron + hexazinone + sulfometuron & $0,00 \mathrm{~b}$ & $0,00 \mathrm{~b}$ & 0,00 \\
\hline amicarbazone & $0,00 \mathrm{~b}$ & $0,00 \mathrm{~b}$ & 0,00 \\
\hline amicarbazone + isoxaflutole ${ }^{1}$ & $2,50 \mathrm{~b}$ & $0,00 \mathrm{~b}$ & 0,00 \\
\hline sulfentrazone & $0,00 \mathrm{~b}$ & $0,00 \mathrm{~b}$ & 0,00 \\
\hline imazapic & $6,25 \mathrm{a}$ & $3,75 \mathrm{a}$ & 0,00 \\
\hline tebuthiuron + isoxaflutole ${ }^{1}$ & $0,00 \mathrm{~b}$ & $0,00 \mathrm{~b}$ & 0,00 \\
\hline F & $11,35^{*}$ & $9,00 *$ & - \\
\hline $\mathrm{CV}(\%)$ & 130,9 & 200,0 & - \\
\hline D.M.S. & 3,02 & 1,98 & - \\
\hline
\end{tabular}

${ }^{1}$ Associação dos herbicidas em aplicação sequencial em pré-emergência da cultura e das plantas daninhas. ${ }^{2}$ Dias após a aplicação. Médias seguidas de mesma letra, nas colunas, não diferem entre si, pelo teste de Tukey (p>0,05). *Significativo a $5 \%$ de probabilidade

\section{Conclusões}

Portanto, pode-se concluir que os herbicidas diuron + hexazinone + sulfometuron $\left(1025+289+24,65\right.$ g i.a.ha $\left.{ }^{-1}\right)$, amicarbazone $\left(1050\right.$ g i.a.ha $\left.{ }^{-1}\right)$, sulfentrazone $\left(800\right.$ g i.a.ha $\left.{ }^{-1}\right)$, e as aplicações sequenciais de amicarbazone $(700$ g i.a.ha $\left.{ }^{-1}\right)$ e isoxaflutole (60 g i.a.ha $\left.{ }^{-1}\right)$, podem ser considerados como excelentes alternativas para o controle de diferentes espécies de cordade-viola (I. hederifolia, I. quamoclit e $i$. nil) quando aplicados em pré-emergência da cultura da cultura da cana-de-açúcar na época seca sem proporcionar fitotoxicidade no desenvolvimento inicial da cultura de cana-deaçúcar na época seca.
Já os tratamentos com o herbicida imazapic (133 g i.a.ha $\left.{ }^{-1}\right)$ e a aplicação sequencial de tebuthiuron (750 g i.a.ha $\left.{ }^{-1}\right)$ e isoxaflutole $\left(60 \mathrm{~g}\right.$ i.a.ha $\left.{ }^{-1}\right)$ podem ser considerados como alternativas para o controle apenas de Ipomoea quamoclit quando aplicados em pré emergência da cultura da cana-de-açúcar na época seca.

\section{Referências}

Azania, C.A.M.; Azania, A.A.P.M.; Pizzo, I.V.; Schiavetto, A.R.; Zera, F.S.; Marcari, M.A. et al. Manejo químico de convolvulaceae e euphorbiaceae em cana-de-açúcar em período 
de estiagem. Planta Daninha, v.27, n.4, p.841848, 2009.

Azania, C.A.M.; Casagrande, A.A.; Rolim, J.C. Seletividade de imazapic às soqueiras de canade-açúcar (Saccharum spp.). Planta Daninha, v.19, n.3, p.345-350, 2001.

Bhullar, M.S.; Walia, U.S.; Singh, S.; Singh, M.; Jhala, A.J. Control of morning-glories (Ipomoea spp.) in sugarcane (Saccharum spp.). Weed Technology, v.26, n.1, p.77-82, 2012.

Carvalho, F.T.; Queiroz, J.R.G.; Toledo, R.E.B. Eficácia do herbicida amicarbazone no controle de cordas-de-viola na cultura da cana-de-açúcar (Saccharum spp.). Revista Brasileira de Herbicida, v.10, n.3, p.183-189, 2011.

Empresa Brasileira de Pesquisa Agropecuária EMBRAPA. Sistema brasileiro de classificação de solos. 3.ed. Brasília, 2013. $353 \mathrm{p}$.

Esquivel, V.A.E.; González, X.R.; Leor, E.N.B. Evaluación de herbicidas residuales para el control de malezas em Guanábana (Annona muricata L.). Revista Chapingo: Serie Horticultura, v.16, n.1, p.5-12, 2010.

Ferreira, D.F. Sisvar: A computer statistical analysis system. Ciência e Agrotecnologia, v.35, n.6, p.1039-1042, 2011.

Griffin, J.L.; Judice, W.A. Winter weed control in sugarcane. Journal of the American Society of Sugar Cane Technologists, v.29, p.128-136, 2009.

Jones, C.A.; Griffin, J.L. Red morning-glory (Ipomoea coccinea) control and competition in sugarcane. Journal of the American Society of Sugar Cane Technologists. v.29, n.1, p.25-53, 2009.

Kissmann, K.G; Groth, D. Plantas infestantes e nocivas. 2.ed. São Paulo: BASF, 1999. Tomo II, 978p.

Kuva, M.A.; Pitelli, R.A.; Alves, P.L.C.A.; Salgado, T.P.; Pavani, M.C.D.M. Banco de sementes de plantas daninhas e sua correlação com a flora estabelecida no agroecossistema cana-crua. Planta Daninha, v.26, n.4, p.735744, 2008.

Kuva, M.A; Pitelli, R.A.; Salgado, T.P. Fitossociologia de comunidades de plantas daninhas em agroecossistema cana-crua. Planta Daninha, v.25, n.3, p.501-511, 2007.

Mancuso, M.A.C.; Negrisoli, E.; Perim, L. Efeito Residual de herbicidas no solo ("Carryover"). Revista Brasileira de Herbicidas, v.10, n.2, p.151-164, 2011.

Miller, P.; Westra, P. Herbicide selectivity and performance. Fort Collins: Colorado State University, Corporative Extension Service. Crop Series Production, 1998. 563p.

Pitelli, R.A. Interferência das plantas daninhas em culturas agrícolas. Informe Agropecuário, v.11, n.129, p.16-27, 1985.

Santos, G.; Francischini, A.C.; Oliveira Neto, A.M.; Guerra, N.; Alonso, D.G.; Dan, H.A. et al. Eficácia e seletividade do herbicida imazapic isolado ou associado a outros herbicidas aplicado com e sem cobertura de palha de canade-açúcar. Revista Brasileira de Herbicidas, v.8, n.3, p.75-84, 2009.

Silva Junior, A.C.; Queiroz, J.R.G.; Martins, D. Quantidade de chuva e lixiviação do herbicida metribuzin através de planta bioindicadora. Revista Brasileira de Engenharia Agrícola e Ambiental, v.19, n.6, p.592-597, 2015a.

Silva Junior, A.C.; Pereira, M.R.R.; Martins, D. Evaluating percolation of the herbicide $\mathrm{S}$ metolachlor with bioindicator plants. Revista de Ciências Agrárias, v.58, n.1, p.36-44, 2015b.

Silva, F.M.L.; Cavalieri, S.D.; Velini, E.D.; Cordeiro, J.G.F.; José, A.R.S. Controle de Urochloa decumbens e Ipomoea triloba pela associação de herbicidas com palha de cana-deaçúcar. Revista Brasileira de Herbicidas, v.10, n.3, p.200-209, 2011.

Sociedade Brasileira Da Ciência Das Plantas Daninhas - SBCPD. Procedimentos para 
instalação, avaliação e análise de experimentos com herbicidas. Londrina: SBCPD, 1995. 42 p.

Toledo, R.B.E; Cason, J.B., Velini, E.D.; Negrisoli, E.; Corrêa, M.R.; Perim, L. et al. DuPont $^{\mathrm{TM}}$ FRONT ${ }^{\circledR}$ : Conceito de Excelência no Controle de Plantas Daninhas na Época Seca. In: Congresso Brasileiro da Ciência das Plantas Daninhas, 27, 2010. Ribeirão Preto. Palestras Inovações Tecnológicas. 2010. CD-ROM.

Toledo, R.E.B.; Negrisoli, E. Biologia e manejo de plantas daninhas em cana-de-açúcar. In: Baldin, E.L. (Ed.) - II SIMPROT - Avanços em Fitossanidade. Botucatu: Unesp/FEPAF, 2011. p. 105 - 120.

Walker, A.; Moon, Y.; Welch. S. J. Influence of temperature, soil moisture and soil characteristics on the persistence of alachlor. Pest Management Science, v.35, n.1, p.109116, 1992. 